\title{
Cell-Assisted Lipotransfer for the Treatment of Parry-Romberg Syndrome
}

\author{
Yanko Castro-Govea ${ }^{1}$, Oscar De La Garza-Pineda ${ }^{1}$, Jorge Lara-Arias ${ }^{2}$, \\ Hernán Chacón-Martínez ${ }^{1}$, Gabriel Mecott-Rivera ${ }^{1}$, Abel Salazar-Lozano ${ }^{1}$, \\ Everardo Valdes-Flores ${ }^{1}$ \\ ${ }^{1}$ Plastic, Reconstructive and Aesthetic Surgery Service, ${ }^{2}$ Bone and Tissue Bank, "Dr. José E. González" University Hospital, Universidad \\ Autónoma de Nuevo Leon, Monterrey, Mexico
}

Progressive facial hemiatrophy, also known as Parry-Romberg syndrome, is a progressive and self-limited deformation of the subcutaneous tissue volume on one side of the face that creates craniofacial asymmetry. We present the case of a patient with a five-year history of progressive right facial hemiatrophy, who underwent facial volumetric restoration using cell-assisted lipotransfer (CAL), which consists of an autologous fat graft enriched with adipose-derived stem cells (ASCs) extracted from the same patient. ASCs have the capacity to differentiate into adipocytes. They also promote angiogenesis, release angiogenic growth factors, and some can survive as stem cells. The use of autologous fat as a filler in soft tissue atrophy has been satisfactory in patients with mild and moderate Parry-Romberg syndrome. Currently, CAL has showed promising results in the long term by decreasing the rate of fat reabsorption. The permanence and stability of the graft in all the injected areas has showed that autologous fat grafts enriched with stem cells could be a promising technique for the correction of defects caused by this syndrome.

Keywords Adipose derived stem/stromal cells / Lipoinjection / Cell therapy / Cell-assisted lipotransfer / Parry-Romberg syndrome
Correspondence: Yanko Castro-Govea Plastic, Reconstructive and Aesthetic Surgery Service, "Dr. José E. González" University Hospital, Universidad Autónoma de Nuevo Leon, Av. Francisco I. Madero \& Av. Gonzalitos s/n, Colonia Mitras Centro, Monterrey 64460, Mexico

Tel: +52-81-83-48-76-56

Fax: +52-81-83-47-61-17

E-mail:goveayanko@hotmail.com

Special thanks to the Bone and Tissues Bank of the Traumatology and Orthopedic Service of the "Dr. José E. González" University Hospital, the Autonomic University of Nuevo Leon, for their help in the cellular processing part of our work.

No potential conflict of interest relevant to this article was reported.

Received: 5 Sep 2012 • Revised: 1 Nov 2012 • Accepted: 5 Nov 2012

pISSN: 2234-6163・ elSSN: 2234-6171 • http://dx.doi.org/10.5999/aps.2012.39.6.659 • Arch Plast Surg 2012;39:659-662

\section{INTRODUCTION}

Progressive facial hemiatrophy, also known as Parry-Romberg syndrome, is a rare disease $(1 / 700,000)$, which usually affects women. It begins in the first two decades of life; however, it has also been reported in the fifth or sixth decade with a mean age of presentation of 8.8 years. It is characterized by a progressive, self-limited deformation and reduction of the subcutaneous tissue volume on one side of the face, which involves scar-like skin changes, atrophy of the subcutaneous and adipose tissue, circum- scribed osteoporosis, trigeminal neuralgia, and changes in the hair and eyes [1].

The first sign of this disease is thinning of the skin and subcutaneous tissue, often preceded by a discoloration of the hair or alopecia areata. It may also involve the mouth, nose or ear, and damage is usually limited to a trigeminal area and it usually does not cross the midline. The final result is a craniofacial asymmetry, which not only causes aesthetic discomfort, but also weakens self-esteem, affecting growth and intellectual development of the patient [2]. 
Throughout history many procedures have been described in an attempt to increase the volume lost by gradual atrophy of soft tissues: dermis-fat grafts, omental free flaps, and musculoskeletal free flaps, just to mention a few. Currently the use of a fat transfer to replenish volume is an increasingly popular method in the field of craniofacial surgery [3]. The introduction of liposuction and liposculpture techniques by Illouz [4] and Fournier and Otteni [5] was a milestone in the history of autologous fat transfer. Subsequently, Coleman [6] indicated that the reabsorption of injected fat is a constant and would require repeated injection. This led him to describe better methods for the aspiration, processing and application of fat, which includes thin cannulas, decantation, centrifugation, and filtration of the lipoaspirate.

Matsumoto et al. [7] described a technique called cell-assisted lipotransfer (CAL) that consists of an autologous adipose tissue transplant enriched with adipose-derived stem cells (ASCs). Using enzymatic digestion with collagenase and centrifugation a cell pellet known as the stromal vascular fraction (SVF) that contains stromal cells, endothelial progenitor cells, ASCs, preadipocytes, and is devoid of adipocytes, is used to enrich the fat graft to be injected.

ASCs from the SVF have several advantages: they can differentiate directly into adipocytes and contribute to adipose tissue regeneration, promote angiogenesis and survival of the graft through differentiation into endothelial cells, release angiogenic growth factors in response to injury and hypoxia and finally, some can survive as stem cells. Yoshimura et al. [8,9] used this technique in patients with facial lipoatrophy and cosmetic breasts augmentation with excellent results.

\section{CASE}

The patient is a 35-year-old man with no previous medical history, diagnosed with progressive right hemifacial atrophy of 10 years evolution and 5 years with a stabilized condition. On examination, alopecia was found in the parietal and temporal region, together with subcutaneous tissue atrophy of the temporal region and the right midface, tooth loss, decreased range of motion of the temporomandibular joint, and trigeminal neuralgia. Computed tomography with 3-dimensional (3D) reconstruction showed

Table 1. Injected volume per facial zone

\begin{tabular}{|lc|}
\hline Facial region & Injected volume $(\mathrm{mL})$ \\
\hline Temporal region & 15 \\
Cheek & 25 \\
Lips & 3 \\
Malar region & 15 \\
Mandibular region and base of the neck & 35 \\
\hline
\end{tabular}

Fig. 1. A 35-year-old male patient diagnosed with Parry-Romberg syndrome

$(A, C, E)$ Preoperative view. (B, D, F) Postoperative view 12 months after lipoinjection enriched with stem cells and elements of the stromal vascular fraction.
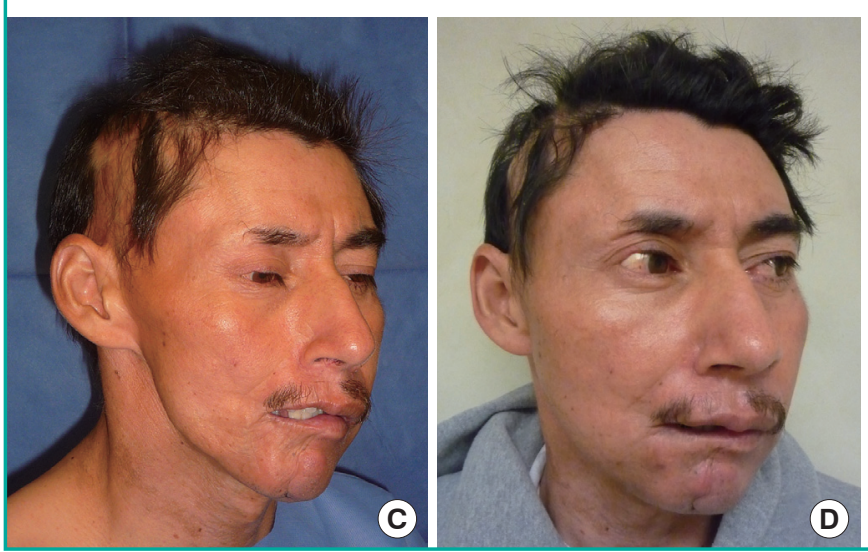
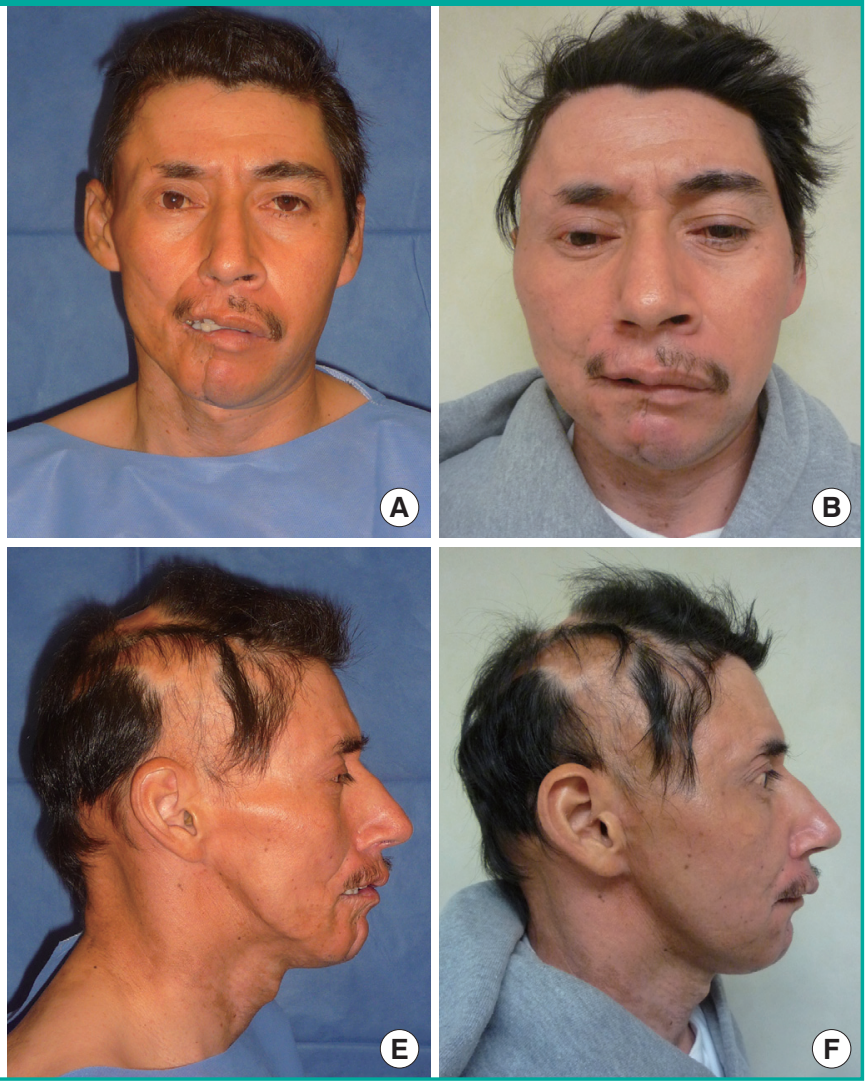
the absence of the temporomandibular joint and a significant reduction of tissue volume in the affected side.

We preformed a previous analysis of the degree of atrophy marking the face with 18 squares using four horizontal planes crossed by seven vertical planes. This helped us identify the areas and volume to inject for facial volume replacement under general anesthesia. We infiltrated the skin with a tumescent Klein solution (solucion HT 1,000 mL with epinephrine $1 \mathrm{mg} / \mathrm{mL}$, PiSA Farmaceutica Mexicana, SA de CV, Guadalajara, Mexico) in the periumbilical region and continued with fat extraction with a 3-mm blunt cannula connected to a $20 \mathrm{~mL}$ syringe. We extracted a total of $300 \mathrm{~mL}$ of fat, from which we separated $150 \mathrm{~mL}$ for processing and extraction of the ASCs. We followed the procedure described by Yoshimura et al. [8,9] (except for the isolation of stem cells from the fluid portion): digestion with collagenase, type II (Gibco, Invitrogen, Carlsbad, CA, USA) and centrifugation $(800 \times \mathrm{g}, 10$ minutes), with a duration of approximately 90 minutes. With the resulting pellet we enriched the $150 \mathrm{~mL}$ initially separated. For injection we used 1 and $3 \mathrm{~mL}$ syringes connected to a 2 -mm blunt cannula; $50 \%$ of the enriched fat was injected into the submuscular layer, and the remaining fat in the subcutaneous stratum (Table 1).

\section{DISCUSSION}

The postoperative evolution of our patient in the short and long term (1 and 12 months) was satisfactory (Fig. 1). Infiltration with enriched autologous fat containing ASCs reduced the severe depression of the frontotemporal region, and provided better volume and symmetry. An acceptable improvement of the malar prominence and cheek was also achieved, with greater volume and projection on the front view and profile. The contour of the mandibular border from the angle to the chin was redefined, achieving a better balance, even the neck base benefitted volumetrically. It is important to point out the permanence of the fat graft in the lips, which allows the teeth to be hidden because of the increase in lip volume. The permanence of the graft remained stable in all the injected areas, even in the nasolabial folds and lips, which are areas of maximum mobility and reabsorption.

Finally, from our point of view and also that of the patient, another important aspect with this technique, which has not been reported in the literature, is the clinical improvement of skin quality: better moisture, texture, shine, and even elasticity.

Soft tissue involvement in Parry-Romberg syndrome is global, including the craniofacial skeleton. It may have varying degrees of atrophy and hence the facial asymmetry is more evident. Although there is no treatment for the underlying etiology, clinical protocols with immunomodulatory drugs are being performed.
Currently, we focus therapy on restoring volume and improving facial expressions.

The literature has reported better results when aspirated fat is processed with Coleman's method, which has less reabsorption and more predictable results. The use of autologous fat as a filler has been quite acceptable in patients with soft tissue atrophy, such as in Parry-Romberg syndrome. For this reason, we propose the combination of autologous fat transplant with SVF elements that contain ASCs. This technique could be considered standard treatment $[10,11]$.

A larger study with more patients will provide the basis for understanding the involvement of ASCs, not only as an adjunct for tissue regeneration, but also for the skin itself. In the near future we consider that its use will be the beginning of a new treatment strategy for patients with different degrees of soft tissue atrophy.

\section{REFERENCES}

1. Terenzi V, Leonardi A, Covelli E, et al. Parry-Romberg syndrome. Plast Reconstr Surg 2005;116:97e-102e.

2. Inigo F, Jimenez-Murat Y, Arroyo O, et al. Restoration of facial contour in Romberg's disease and hemifacial microsomia: experience with 118 cases. Microsurgery 2000;20: 167-72.

3. Guerrerosantos J, Guerrerosantos F, Orozco J. Classification and treatment of facial tissue atrophy in Parry-Romberg disease. Aesthetic Plast Surg 2007;31:424-34.

4. Illouz YG. Surgical remodeling of the silhouette by aspiration lipolysis or selective lipectomy. Aesthetic Plast Surg 1985; 9:7-21.

5. Fournier PF, Otteni FM. Lipodissection in body sculpturing: the dry procedure. Plast Reconstr Surg 1983;72:598-609.

6. Coleman SR. Facial recontouring with lipostructure. Clin Plast Surg 1997;24:347-67.

7. Matsumoto D, Sato K, Gonda K, et al. Cell-assisted lipotransfer: supportive use of human adipose-derived cells for soft tissue augmentation with lipoinjection. Tissue Eng 2006; 12:3375-82.

8. Yoshimura K, Sato K, Aoi N, et al. Cell-assisted lipotransfer for facial lipoatrophy: efficacy of clinical use of adipose-derived stem cells. Dermatol Surg 2008;34:1178-85.

9. Yoshimura K, Sato K, Aoi N, et al. Cell-assisted lipotransfer for cosmetic breast augmentation: supportive use of adipose-derived stem/stromal cells. Aesthetic Plast Surg 2008;32:48-55.

10. Sterodimas A, Huanquipaco JC, de Souza Filho S, et al. Autologous fat transplantation for the treatment of ParryRomberg syndrome. J Plast Reconstr Aesthet Surg 2009;62: 
e424-6.

11. Koh KS, Oh TS, Kim H, et al. Clinical application of human adipose tissue-derived mesenchymal stem cells in progressive hemifacial atrophy (Parry-Romberg disease) with mi- crofat grafting techniques using 3-dimensional computed tomography and 3-dimensional camera. Ann Plast Surg 2012; 69:331-7. 